\title{
Decentralizing Oxygen Availability and Use at Primary Care Level for Children Under-Five with Severe Pneumonia an Experience from 12 Health Centers in Ethiopia: A Pre-Post Non-Experimental Study Design
}

Habtamu Seyoum Tolla

Clinton Health Access Initiative

Dinkineh Bikila Woyessa

Clinton Health Access Initiative

Rahel Belete Balkewu

Clinton Health Access Initiative

Yigeremu Abebe Asemere

Clinton Health Access Initiative

Zinabie Feleke Fekadu

Clinton Health Access Initiative

Alemayehu Belete

Clinton Health Access Initiative

Martha Gartley

Clinton Health Access Initiative

Audrey Battu

Clinton Health Access Initiative

Felix Lam

Clinton Health Access Initiative

Alebel Yaregal Desale ( $\nabla$ alebel.yaregal@yahoo.com )

Clinton Health Access Initiative

Research Article

Keywords:

Posted Date: January 31st, 2022

DOI: https://doi.org/10.21203/rs.3.rs-1220851/v1 
License: (c) (i) This work is licensed under a Creative Commons Attribution 4.0 International License. Read Full License 


\section{Abstract}

Pneumonia is the leading infectious cause of death in children worldwide, accounting for $15 \%$ of all deaths in children under the age of five. Hypoxemia is a major cause of death in patients suffering from pneumonia. There is strong evidence that using pulse oximetry and having reliable oxygen sources in health care facilities can reduce deaths due to pneumonia by one-third. Despite its importance, hypoxemia is frequently overlooked in resource-constrained settings. Aside from the limited availability of pulse oximetry, evidence showed that healthcare workers did not use it as frequently to generate evidence-based decisions on the need for oxygen therapy. As a result, the goal of this study was to assess the availability of medical oxygen devices, operating manuals, guidelines, healthcare workers' knowledge, and skills in the practice of hypoxemia diagnosis and oxygen therapy in piloted health centers of Ethiopia. A pre-post non-experimental study design was employed. An interviewer-administered questionnaire was used to collect primary data and review medical record charts. A chi-square test with a statistical significance level of $\mathrm{P}<0.05$ was used as a cut-off point for claiming statistical significance. Eighty-one percent of healthcare workers (HCWs) received oxygen therapy training, up from $6 \%$ at baseline. As a result of the interventions, the knowledge for example pulse oximetry uses and oxygen therapy provision, skill such as proper reading and interpretation of the reading of saturation and pulse, and practice of oxygen therapy has significantly improved among health care workers in the piloted HCs. In terms of availability of oxygen supply devices (cylinder, concentrator, and pulse oximetry) in the facilities, more than half of the facilities $7(58 \%)$ did not have any at baseline, but due to the interventions, all facilities were equipped with the oxygen devices. Given the prevalence of pneumonia and hypoxemia, a lack of access to oxygen delivery devices, as well as a lack of knowledge and skill among HCWs in the administration of oxygen therapy, may represent an important and reversible barrier to improved child survival. Therefore, scaling up clinician training, technical support, availability of oxygen devices, guidelines, manuals, strengthening maintenance schemes, and close monitoring of HCWs and health facilities is strongly advised.

\section{Introduction}

Pneumonia is the single most common infectious cause of death in children, accounting for $15 \%$ of all deaths in children under the age of five1. Hypoxemia, or a low level of oxygen in the blood, is a major fatal complication of pneumonia, and the risk of death increases as the severity of hypoxemia increases 2-5. According to a systematic review, 13.3 percent of children with pneumonia have hypoxemia 6. Furthermore, according to the UN IGME report, 23 percent of the 5.9 million annual child deaths are caused by neonatal conditions such as birth asphyxia, sepsis, and low birth weight, all of which can lead to hypoxemia4. Clinical manifestations of hypoxemia are insensitive7,8. Many children with hypoxemia are missed if pulse oximetry is not used on a regular basis7,9.

Countries are gaining experience in the clinical, biomedical, and training aspects of establishing and maintaining effective oxygen delivery systems in hospitals and lower-level health facilities ${ }^{4,10}$. There is evidence that the use of pulse oximetry and the availability of reliable oxygen sources in district hospitals 
can reduce pneumonia death rates by about one-third ${ }^{4,10}$. Similarly, improving access to oxygen devices (oxygen concentrator and cylinder) and pulse oximetry has been shown to reduce childhood pneumonia mortality by up to $35 \%$ in high-burden child pneumonia settings ${ }^{5,9}$.

Despite its importance in almost all types of severe illness, hypoxemia is frequently under-recognized and under-managed in resource-constrained settings. For a significant number of critically ill children admitted to hospitals in low-resource settings, oxygen therapy remains an unattainable luxury ${ }^{4}$. An assessment report in Ethiopia revealed that functional oxygen service availability and pulse oximetry use are very low, with only $2 \%$ of Health Centers (HCs) having a fully functional cylinder and/or concentrator available, and no functional pulse oximeter available in 314 (100\%) of the health centers visited for the assessment ${ }^{11}$. This is especially true for patients in lower-level health facilities, such as HCs, where supplies are frequently unreliable and treatment benefits may be unavailable or diminished due to poorly maintained, inappropriate equipment, poorly trained staff, or inadequate guidelines ${ }^{4}$.

Raising the knowledge and skill on these issues is likely to have a significant impact on clinical and public health practices. Health professionals should be cognizant of the clinical signs and symptoms that indicate the presence of hypoxemia. More widespread use of pulse oximetry, as a noninvasive measure of arterial oxygen saturation, could lead to more reliable detection of hypoxemia. Oxygen therapy must be widely available; even in remote areas, this can be accomplished through the use of oxygen concentrators, which can run on either regular or alternative power sources ${ }^{4}$.

However, evidence showed that, in addition to the limited availability of pulse oximetry in health facilities, Health Care Workers (HCWs) did not use it as frequently as for children with severe pneumonia and thought it was less important for diagnosis than other tools in developing-country clinical settings 312 . Furthermore, to the best of our knowledge, there is no published evidence about its availability and practice at the lower-level facilities (health center) in Ethiopia, despite the fact that $\mathrm{HC}$ is a critical component of the primary health care unit, with each serving an estimated total and under-five population of 25,000 and 4,000 respectively. Therefore, an estimated 48,000 under-five children served in these piloted HCs. It also offers critical child health services such as Integrated Management of Newborn and Child Health Illnesses (IMNCI). According to unpublished reports, oxygen concentrators and pulse oximetry are only available and used at the hospital level. As a result, the goal of this study is to assess the availability of medical oxygen devices, investigate the knowledge and skills of HWs in diagnosing hypoxemia and provide medical oxygen therapy in 12 piloted HCs in Ethiopia.

\section{Methods}

To assess the availability of medical oxygen devices, assess the knowledge, skill and practice of health care workers in the application and use of oxygen therapy baseline assessment was conducted in 12 health centers ( $\mathrm{HCs}$ ) found in four agrarian regions of Ethiopia. Based on the assessment, baseline values were set and interventions such as oxygen concentrator, pulse oximetry, oxygen therapy manuals 
for children and adults, oxygen flow-chart algorithms, oxygen therapy trainings and supportive supervision were provided.

\section{Study design, period, setting and population}

The study employed a pre-post survey design. The study was carried out in 12 health centers located in four agrarian regions of Ethiopia, namely Amhara, Oromia, SNNP, and Tigray, from February to September 2019. The baseline data were collected in February 2019, followed by consecutive months of technical support and mentorship data collection were conducted in May and September 2019. The study setting consisted of 12 high-volume/patient load HCs that were purposefully chosen to meet the geographical equity of HCs in their respective regions. Additionally, HCWs working in the piloted HCs were the study populations. Furthermore, medical record reviews of children aged 0 to 59 months with severe pneumonia cases were included in the study population.

\section{Sample size and sampling procedures}

Censuses of all piloted HCs were considered because the number of piloted HCs were insufficient to apply the sampling procedure. The previous four months of medical records were reviewed retrospectively beginning from the date of the visit back and including all pneumonia cases within that timeframe to see how the cases were managed in the process of oxygen administration prior to the provision of any device, training, and mentorship to set baseline values. Following the baseline assessment, the pilot facilities received the necessary training on hypoxemia diagnosis and oxygen therapy in children based on a WHOadopted national training manual. All HCs were given pulse oximetry and oxygen concentrators. On-site mentoring was also offered to improve HCWs knowledge and skills. Medical records of All severe pneumonia cases were reviewed twice, in every three-month interval retrospectively. A total of 2,960 medical record charts have been reviewed since the beginning. Furthermore, since baseline, $36 \mathrm{HCWs}$ knowledge and skills have been assessed prospectively.

\section{Data collection methods and tools}

A mix of quantitative and qualitative data collection techniques were used to collect primary data from HCWs and secondary data from medical record reviews. The methods listed below were used to collect data. To collect the variables of interest, structured data extraction tools were created. SurveyCTO (Dobility Inc, Cambridge, MA USA), an electronic data collection software, was programmed with the data collection tools, and the software application was downloaded onto tablet computers.

\section{Medical record review}

The review of medical records were conducted using standard, structured, and closed-ended questionnaires based on WHO guidelines ${ }^{4}$. In every three months, chart reviews were performed to assess the status of medical oxygen therapy for pediatric patients. Baseline values were set by reviewing medical records retrospectively. Following the implementation of the interventions, medical records were 
also reviewed twice. Because the volume of cases was too small to sample during the review of medical records, all severe pneumonia cases identified in that specific $\mathrm{HC}$ during the review period were included. Following that, the procedures and applications of oxygen therapy were evaluated, including oxygen check-ups, saturation records, oxygen prescriptions if hypoxemic, flow rate, routes of administration, follow-up status, and the child's outcomes. Technical support and feedback were provided to HCWs based on the gaps identified in the chart reviews. The reviewers of the medical records were health care professionals who have a minimum of a second degree in health science and have a wealth of experience in the field.

\section{Interview}

To assess HCW knowledge and skills, the interview guide used both closed and open-ended questionnaires. The interview guide includes questions about their pulse oximetry knowledge, skill, and application, as well as their use of oxygen concentrators. Respondents for the key informant interview (KII) were purposefully chosen based on their specific roles. Eligible respondents for the KII were initially identified in health facilities and contacted, with the purpose of the study explained. At the baseline assessment, the interviewees were health professionals assigned to oxygen treatment units.

\section{Observations}

The observation method was also used to assess HCWs' skill and practice on volunteers because it can be empirically calibrated by observations on normal volunteers ${ }^{13}$. This reinforces and aids in the triangulation of data gathered through chart reviews and key informant interviews.

\section{Interventions}

Based on the baseline assessment, oxygen devices such as oxygen concentrators and pulse oximetry were procured and provided. Moreover, oxygen therapy manuals for children and adults, oxygen flowchart algorithms, oxygen therapy trainings for clinicians, supportive supervisions and technical support were provided. Similarly, to ensure the availability of medical oxygen, device maintenance system was designed in the context of primary health care units which enables them to easily link with biomedical engineers and technicians available either at woreda health office, zone health department, regional health bureau, or nearby hospitals.

\section{Data Analysis}

MS-Excel Office 2016 was used to clean the data. Statistical Packages for Social Sciences were also used for additional cleaning and preparation (SPSS). The primary outcomes of the study were as follows: (1) the proportion of children under the age of five admitted with pneumonia who had a SpO2 measurement; (2) the proportion of children under the age of five admitted with severe pneumonia and with $\mathrm{SpO} 2<93 \%$ that received oxygen; (3) the proportion of facilities with an oxygen concentrator and a pulse oximeter; and (4) the knowledge and skills of HCWs on oxygen therapy practice. To present key 
findings, a descriptive analysis was used to generate frequencies, tables, and charts. Chi-squared tests were used to determine whether there were statistical differences between consecutive follow-up periods. A p-value of less than 0.05 was used as a cut-off point to determine whether or not there was a relationship.

\section{Ethical Clearance}

The research protocol was reviewed, and ethical approval was obtained from the Ethiopian Public Health Institute (EPHI) under protocol number EPHI-IRB-175-2019, with a letter of approval dated 19 June 2019 and referencing EPHI13.6/136. A confirmation/clearance letter was also obtained from the Ministry of Health (MoH) and Regional Health Bureaus (RHBs). All participants in the study gave their verbal consent. There was no known risk in the study, and no payment was given to participants.

\section{Results}

\section{Health facilities background information}

The piloted HCs found in four agrarian regions. The distance between the $\mathrm{HC}$ and the nearest referral hospital ranges from 1 to 60 kilometers. During the study period, 516,266 people visited the outpatient department (OPD). Out of the total OPD visitors, 64,398 (12.5\%) were children under the age of five; among these under-five visitors, 3,893 (6\%) and $267(0.41 \%)$ were pneumonia and severe pneumonia cases, respectively. The average number of health professionals assigned to work in the outpatient departments of piloted HCs were 2.25 per HC (Figure 1).

In terms of the availability of oxygen supply devices (cylinder, concentrator, and pulse oximetry) in the facilities, more than half of the facilities $7(58 \%)$ did not have any at baseline, but due to the interventions, all facilities were equipped with the oxygen devices. Since the beginning, a total of 2,960 medical records have been reviewed. Of the total medical records, 58 percent and 42 percent were male and female respectively. In general, $240(8 \%), 1203(41 \%)$, and $1513(51 \%)$ of the total number of medical records reviewed fall within the age category of $<=1$ month, $>1-<=11$ months, $>11-59$ months and 4 missing data (Table 1).

\section{Knowledge Assessment}

The majority (94\%) of professionals in the piloted health facilities did not have oxygen therapy training at baseline, and as part of our designed interventions, 29 (81\%) of HCWs received oxygen therapy training in the piloted health facilities.

Based on HCWs' knowledge assessment of whether clinical signs are reliable predictors of hypoxemia or not, our study revealed that only $14 \%$ of HCWs correctly answered at baseline. However, after training and technical assistance, the majority $30(83 \%)$ of them responded that clinical signs are not a reliable predictor of hypoxemia. Furthermore, when HCWs were asked whether hypothermia and poor peripheral 
perfusion would affect the saturation measurement of pulse oximetry, the majority $34(94 \%)$ correctly responded. However, only $19 \%$ of them were able to respond correctly at the start.

In terms of HCWs' knowledge of the cut-off point of oxygen saturation (SPO2) to initiate oxygen therapy, 9(25\%), 34(94.4\%), and 35(97\%) correctly stated that the cut-off points at baseline (February 2019), May 2019, and end-line (September 2019) respectively. Because of ongoing capacity building, the majority of $\mathrm{HCWs}$ were aware of the recommended cut-off point $(\mathrm{SpO} 2<93 \%)$ for initiating oxygen therapy. As a result, the intervention resulted in a significant improvement over the baseline finding (Figure 2).

The study revealed that after the intervention measures were implemented, oxygen therapy services increased dramatically and were available in all $12 \mathrm{HCs}$. However, only $4(33 \%)$ of the HCs were able to provide oxygen therapy services at the outset. Similarly, the majority $(97 \%)$ of HCWs across all regions used pulse oximetry to measure oxygen saturation, compared to only $17 \%$ at baseline. The provision of oxygen therapy services in HCs and the use of pulse oximetry among HCWs yielded statistically significant $(p<0001)$ results (Figure 3$)$.

The study also assessed HCWs' knowledge of the standard flow rate of oxygen administration for infants (1-2LPM), with the majority (78\%) correctly responding that the standard flow rate which was only $14 \%$ at baseline, it also yields a statistically significant result $(p<0001)$. HCWs who misunderstood the standard flow rate of oxygen administration for infants, on the other hand, sharply declined from the baseline, as shown in the line graph (Figure 4).

\section{Skill Assessment}

According to the demonstration on skill assessment of HCWs, all HCWs were able to properly place the pulse oximetry probe on the finger after training and subsequent technical support, but it was only $5(14 \%)$ at baseline. Similarly, based on the skill assessment for the application of pulse oximetry, all HCWs were able to demonstrate and properly wait until the wave became regular, but only $38 \%$ of them were able to wait until the wave became regular at baseline. Additionally, findings revealed that all HCWs were able to correctly read the saturation and pulse based on the mentorship provided on the identified gaps. However, only $5(14 \%)$ of HCWs were able to correctly read the saturation and pulse at baseline. Similarly, appropriate reading interpretation increased significantly from $3 \%$ at baseline to $100 \%$ in subsequent rounds of capacity building and mentorship support. (Figure 5).

Our study also depicted that the majority of HCWs $26(72 \%)$ were unable to demonstrate nasal prong placement at baseline, but with the technical and capacity-building supports provided, all HCWs were able to properly demonstrate. Similarly, almost all HCWs 35(97\%) were unable to properly set the flow rates for nasal prongs at baseline, but this was reversed after the intervention was implemented, and all HCWs were able to properly set flow rates.

\section{The practice of Oxygen Therapy}


According to our findings, HCWs' practice of recording oxygen saturation has significantly improved. The practice of recording oxygen saturation levels, as shown in the bar graph below, shows progressive improvement following interventions. Although oxygen saturation record was zero at the start, the status of the oxygen saturation (SPO2) record for pediatric patients at diagnosis (at triage and/or at initial assessment) increased from $0-66 \%$ as a result of capacity building training and technical support to HCWs. (Figure 6).

The findings revealed that $420(37 \%)$ of the total records reviewed for the respiratory rate at diagnosis were incomplete and considered information missing at baseline. The respiratory rates of $111(11 \%)$ and $171(20 \%)$ of the cases were not recorded after the intervention in the consecutive follow-up. Overall, there were improvements when compared to the baseline, but there were still some fluctuations.

In terms of the status of oxygen saturation (SPO2) record for patients at diagnosis (at triage and/or at initial assessment), the oxygen saturation was not recorded at all at baseline, but it was recorded for 388 $(40 \%)$ and $569(66 \%)$ in the consecutive follow-ups and technical supports, respectively. Of the children whose oxygen saturation levels were measured, 68 and 55 were hypoxemic. The result is displayed in (Figure 7)

\section{Discussion}

The study's findings revealed a lack of oxygen devices and capacity for oxygen therapy across all 12HCs. In almost all facilities, HCWs' abilities to provide oxygen therapy services were severely limited, with significant gaps in knowledge and skill in the use of oxygen concentrators and pulse oximetry. As a result of the identified gaps, oxygen therapies for clinicians were provided, increasing the availability of trained HCWs from $6-81 \%$. In addition to the availability of trained HCWs, their knowledge of the application of oxygen therapy and pulse oximetry use increased from $33-100 \%$ and $17-97 \%$ on the piloted HCs, respectively.

Researchers discovered that low knowledge and skills among HCWs were almost universally reported and that many HCWs had misconceptions and fears about oxygen therapy, which hampered their motivation. The researchers also stated that the projects provided initial training and demonstrated that HCWs' knowledge and skills had improved ${ }^{14-18}$. Furthermore, researchers documented that a lack of guidelines, equipment, and technical difficulties affect HCW motivation and that these challenges can be addressed through on-site training and regular on-site support ${ }^{14,1519-21}$. In line with this, our study identified knowledge and skill gaps among HCWs and provided interventions such as oxygen equipment, on-site training, and regular mentorship, which significantly improved HCWs' knowledge and skill in providing oxygen therapy services in the facilities. Furthermore, the HCWs stated in a qualitative interview that the interventions reduced unnecessary referrals to nearby district hospitals.

A study that looked at the barriers to using oxygen and pulse oximetry in children with pneumonia found that lack of training and guidelines were two of the most frequently cited barriers ${ }^{7}$. Similarly, our study 
found that the majority of HCWs lacked knowledge and skills in using oxygen devices and pulse oximetry at the outset. Furthermore, our baseline study discovered that nearly three-quarters of HCWs believe clinical signs are a reliable predictor of hypoxemia, despite numerous evidences $7,8,22,23$. As a result, interventions such as training and technical support were provided to improve the quality of services and, ultimately, the health outcomes of pediatric patients. These interventions reversed HCWs' misperceptions that clinical signs are reliable predictors of hypoxemia and significantly increased their knowledge from $10(28 \%)$ to 30 (83 percent).

\section{Limitation}

Although the HCs were chosen from four regions of Ethiopia, they were not chosen at random; rather, they were chosen based on patient caseload. Second, the sample sizes could be unrepresentative. As a result, our findings should be applied with caution to HCs with similar settings.

\section{Conclusions}

Due to the effects of the multi-faceted interventions, the availability of medical oxygen devices, oxygen therapy manuals for children and adults and oxygen flow-chart algorithms have significantly changed from the baseline values. Similarly, the knowledge and skill of health care workers in proper use and practice of medical oxygen therapy has significantly improved in the piloted HCs.

In general, our findings can help practitioners and policymakers understand how to improve oxygen therapy in $\mathrm{HC}$ settings, as well as lower level and key components of primary care units that are expected to provide comprehensive child health services like IMNCl. Given the prevalence of pneumonia and hypoxemia, a lack of access to oxygen delivery devices, as well as a lack of knowledge and skill among HCWs in the administration of oxygen therapy, may represent an important and reversible barrier to improved child survival. Scaling up training, technical support, and close monitoring of HCWs and health facilities is therefore strongly advised.

\section{Declarations}

\section{Ethical approval and consent to participate}

Ethical approval was obtained from Ethiopian Public Health Institute with EPHI-IRB meeting No. 042 and protocol number: EPHI-IRB-175-2019

\section{Authors reporting experiments on humans/human data}

All methods were carried out in accordance with relevant guidelines and regulations and in addition to ethical approval from IRB verbal informed consent was obtained from all subjects.

\section{Consent for publication}


Not applicable

\section{Availability of data and materials}

All data generated or analyzed during this study are included in this published article and its supplementary information files.

\section{Competing interest}

The authors declare that no commercial or financial relationships existed that could be interpreted as a potential conflict of interest during the research.

\section{Funding}

Accelerating Access to Lifesaving Child Health Commodities and Services in Ethiopia is a Child Survival program funded by the Bill \& Melinda Gates Foundation under cooperative agreement Investment ID: INV009817 / OPP1133423. The Clinton Health Access Initiative, in collaboration with the local government, is implementing the program. The funder had no involvement in the study's design, data collection, analysis, or manuscript writing.

\section{Authors' contributions}

HabtamuSeyoumTolla, Alebel Yaregal Desale, DinkinehBikilaWoyessa, and ZinabieFelekeFekadu have managed the design of project concepts, methods, managing the process of implementation, drafting and finalization of the manuscript. Alebel Yaregal Desale is also involved in data cleaning and analysis. The rest of the authors have been engaged in the process of project implementation as well as in reviewing the manuscript.

\section{Acknowledgments}

The investigators are grateful to all participants for their assistance with data collection. The Bill \& Melinda Gates Foundation's generous support made this study, availability of medical oxygen and pulse oximetry, and clinical practice of oxygen therapy possible.

\section{References}

1. WHO-Fact sheet. Published online 2019.

2. Herbert LJ, Wilson IH. Pulse oximetry in low-resource settings. Breathe. 2012;9(2):90-97. doi:10.1183/20734735.038612

3. Ginsburg AS, Van cleve WC, Thompson MIW, English M. Oxygen and pulse oximetry in childhood pneumonia: A survey of healthcare providers in resource-limited settings. J Trop Pediatr. 2012;58(5):389-393. doi:10.1093/tropej/fmr103 
4. World Health Organization. Oxygen therapy for children. Oxyg Ther Child a Man Heal Work. Published online 2016:57. http://apps.who.int/iris/bitstream/10665/204584/1/9789241549554_eng.pdf

5. Minstry of Health of Ethiopia. National Medical Oxygen and Pulse Oximetry Scale Up Road Map (2016-2020/21). 2016;(September).

6. Saha S, Hasan M, Kim L, et al. Epidemiology and risk factors for pneumonia severity and mortality in Bangladeshi children $<5$ years of age before 10-valent pneumococcal conjugate vaccine introduction. BMC Public Health. 2016;16(1):1-12. doi:10.1186/s12889-016-3897-9

7. Ginsburg AS, Van cleve WC, Thompson MIW, English M. Oxygen and pulse oximetry in childhood pneumonia: A survey of healthcare providers in resource-limited settings. J Trop Pediatr. 2012;58(5):389-393. doi:10.1093/tropej/fmr103

8. Ayieko P, English M. Europe PMC Funders Group Case Management of Childhood Pneumonia in Developing Countries. 2009;26(5):432-440. doi:10.1097/01.inf.0000260107.79355.7d.Case

9. Duke T, Graham SM, Cherian MN, et al. Oxygen is an essential medicine: A call for international action. Int J Tuberc Lung Dis. 2010;14(11):1362-1368.

10. Duke T, Wandi F, Jonathan M, et al. Improved oxygen systems for childhood pneumonia: a multihospital effectiveness study in Papua New Guinea. Lancet (London, England). 2008;372(9646):1328-1333. doi:10.1016/S0140-6736(08)61164-2

11. Accelerating Policy Change, Translation and Implementation for Pneumonia and Diarrhea Commodities in Ethiopia ,. Report. Published online 2016.

12. Wilcox T, Hirshkowitz A. NIH Public Access. 2015;85(0 1):1-27. doi:10.1016/j.neuroimage.2013.08.045.The

13. Mannhardt F, De Leoni M, Reijers HA. Heuristic mining revamped: An interactive, data-Aware, and conformance-Aware miner. CEUR Workshop Proc. 2017;1920(June 2010):358-359. doi:10.3399/bjgp11X572553

14. Graham H, Tosif S, Gray A, et al. Providing oxygen to children in hospitals: a realist review. 2017; (December 2016):288-302.

15. Nabwire J, Namasopo S, Hawkes M. Oxygen Availability and Nursing Capacity for Oxygen Therapy in Ugandan Paediatric Wards. 2018;(May 2017):97-103. doi:10.1093/tropej/fmx033

16. Gray AZ, Morpeth $M$, Duke T, et al. Improved oxygen systems in district hospitals in Lao PDR: A prospective field trial of the impact on outcomes. BMJ Paediatr Open. 2017;1(1). doi:10.1136/bmjpo2017-000083

17. Bakare AA, Graham H, Ayede Al, et al. Providing oxygen to children and newborns: A multi-faceted technical and clinical assessment of oxygen access and oxygen use in secondary-level hospitals in southwest Nigeria. Int Health. 2019;12(1):60-68. doi:10.1093/inthealth/ihz009

18. Graham H, Tosif S, Gray A, et al. Bulletin of the World Health Organization Providing oxygen to children in hospitals: a realist review. 2017;95(4):1-20. 
19. Fyyaz S. P249 Could an Intronic SNP in the Alpha-1-Antitrypsin Gene Confer Protection to Chronic Obstructive Pulmonary Disease? Thorax. 2012;67(Suppl 2):A173.2-A174. doi:10.1136/thoraxjnl2012-202678.310

20. Enarson P, Vincente L, Gie R, Chokani C. Implementation of an oxygen concentrator system in district hospital paediatric wards throughout Malawi. 2008;048017(February). doi:10.2471/BLT.07.048017

21. Masroor R, Iqbal A, Buland K, Kazi WA. Use of a portable oxygen concentrator and its effect on the overall functionality of a remote field medical unit at 3650 meters elevation. Anaesthesia, Pain Intensive Care. 2013;17(1):45-50.

22. Duke T, Mgone J, Frank D. Hypoxaemia in children with severe pneumonia in Papua New Guinea. Int $J$ Tuberc lung Dis Off J Int Union against Tuberc Lung Dis. 2001;5(6):511-519.

23. Duke T, Blaschke AJ, Sialis S, Bonkowsky JL. Hypoxaemia in acute respiratory and non-respiratory illnesses in neonates and children in a developing country. Arch Dis Child. 2002;86(2):108-112. doi:10.1136/adc.86.2.108

\section{Tables}


Table 1

Medical record review by region and health center

\begin{tabular}{|c|c|c|c|c|c|c|c|c|}
\hline \multirow[t]{2}{*}{ Name of $\mathrm{HC}$} & \multicolumn{4}{|l|}{ Region } & \multirow[t]{2}{*}{ Total } & \multirow{2}{*}{$\begin{array}{l}\text { Baseline } \\
\text { (Feb- } \\
\text { 2019) }\end{array}$} & \multirow{2}{*}{$\begin{array}{l}\text { May } \\
2019\end{array}$} & \multirow{2}{*}{$\begin{array}{l}\text { End-line } \\
\text { (September } \\
\text { 2019) }\end{array}$} \\
\hline & Amhara & Oromia & SNNPR & Tigray & & & & \\
\hline $\begin{array}{l}\text { Kombolcha } \\
\text { HC }\end{array}$ & 250 & & & & 250 & 103 & 48 & 99 \\
\hline Woreta HC & 429 & & & & 429 & 257 & 85 & 87 \\
\hline $\begin{array}{l}\text { Bahir Dar } \\
\text { HC }\end{array}$ & 265 & & & & 265 & 116 & 73 & 76 \\
\hline $\begin{array}{l}\text { Alemgena } \\
\mathrm{HC}\end{array}$ & & 116 & & & 116 & 23 & 44 & 49 \\
\hline $\begin{array}{l}\text { Chefe } \\
\text { Donsa HC }\end{array}$ & & 298 & & & 298 & 96 & 97 & 105 \\
\hline Sebeta HC & & 242 & & & 242 & 103 & 75 & 64 \\
\hline $\begin{array}{l}\text { Millenium } \\
\text { HC }\end{array}$ & & & 93 & & 93 & 38 & 33 & 22 \\
\hline $\begin{array}{l}\text { Wondogenet } \\
\mathrm{HC}\end{array}$ & & & 118 & & 118 & 22 & 60 & 36 \\
\hline Yirgalem HC & & & 234 & & 234 & 67 & 121 & 46 \\
\hline $\begin{array}{l}\text { Edaga } \\
\text { Hamus HC }\end{array}$ & & & & 44 & 44 & 13 & 21 & 10 \\
\hline Megab HC & & & & 298 & 298 & 83 & 131 & 84 \\
\hline Wukro HC & & & & 573 & 573 & 202 & 191 & 180 \\
\hline Total & 944 & 656 & 445 & 915 & 2960 & 1123 & 979 & 858 \\
\hline
\end{tabular}

Figures 


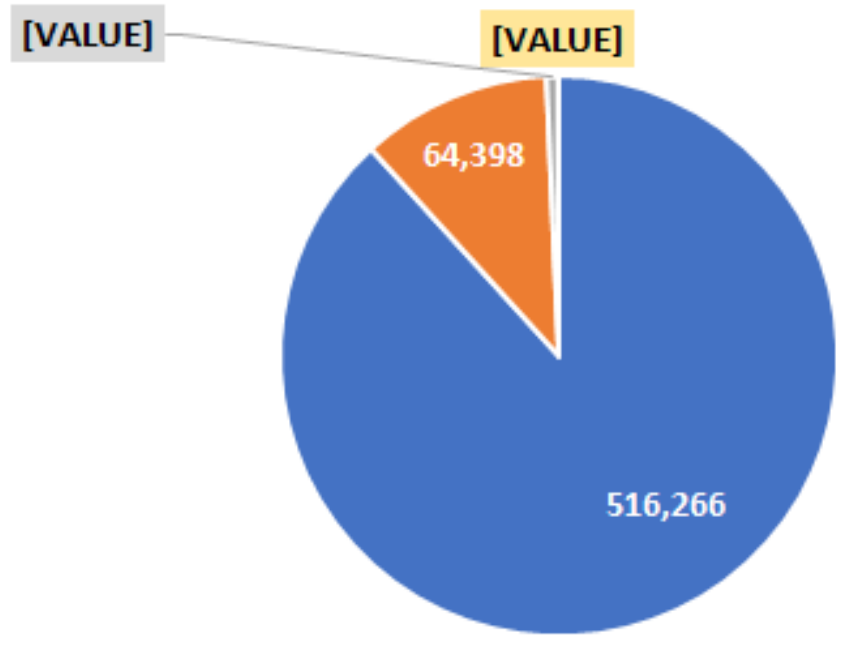

- Total OPD visitors $\quad$ Under 5 children $\quad$ Pneumonia $=$ Severe Pneumonia

\section{Figure 1}

Disaggregation of outpatient visitors and cases

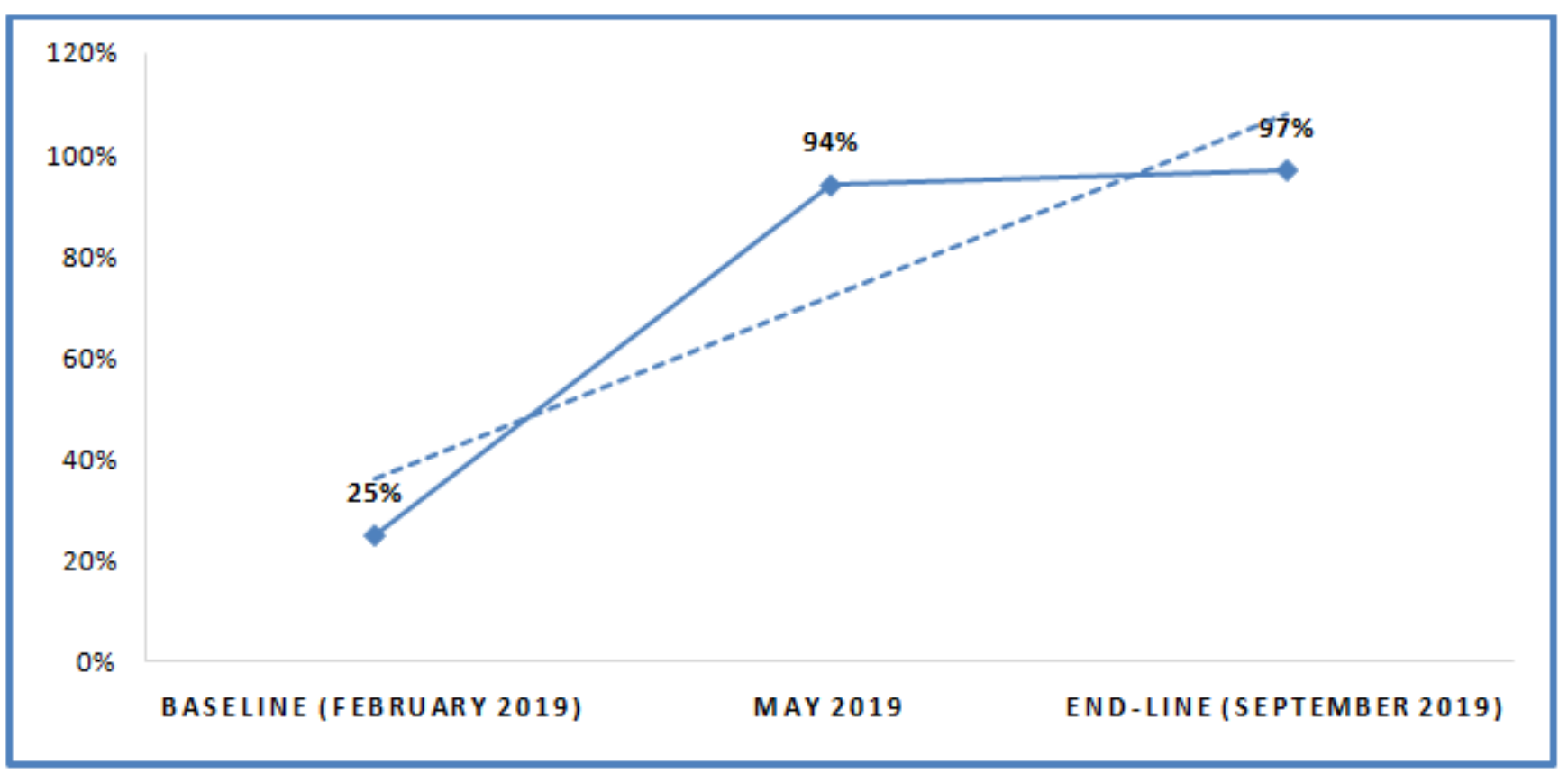

Figure 2

Knowledge of HCWs on the cut-off point of oxygen saturation to initiate oxygen therapy 


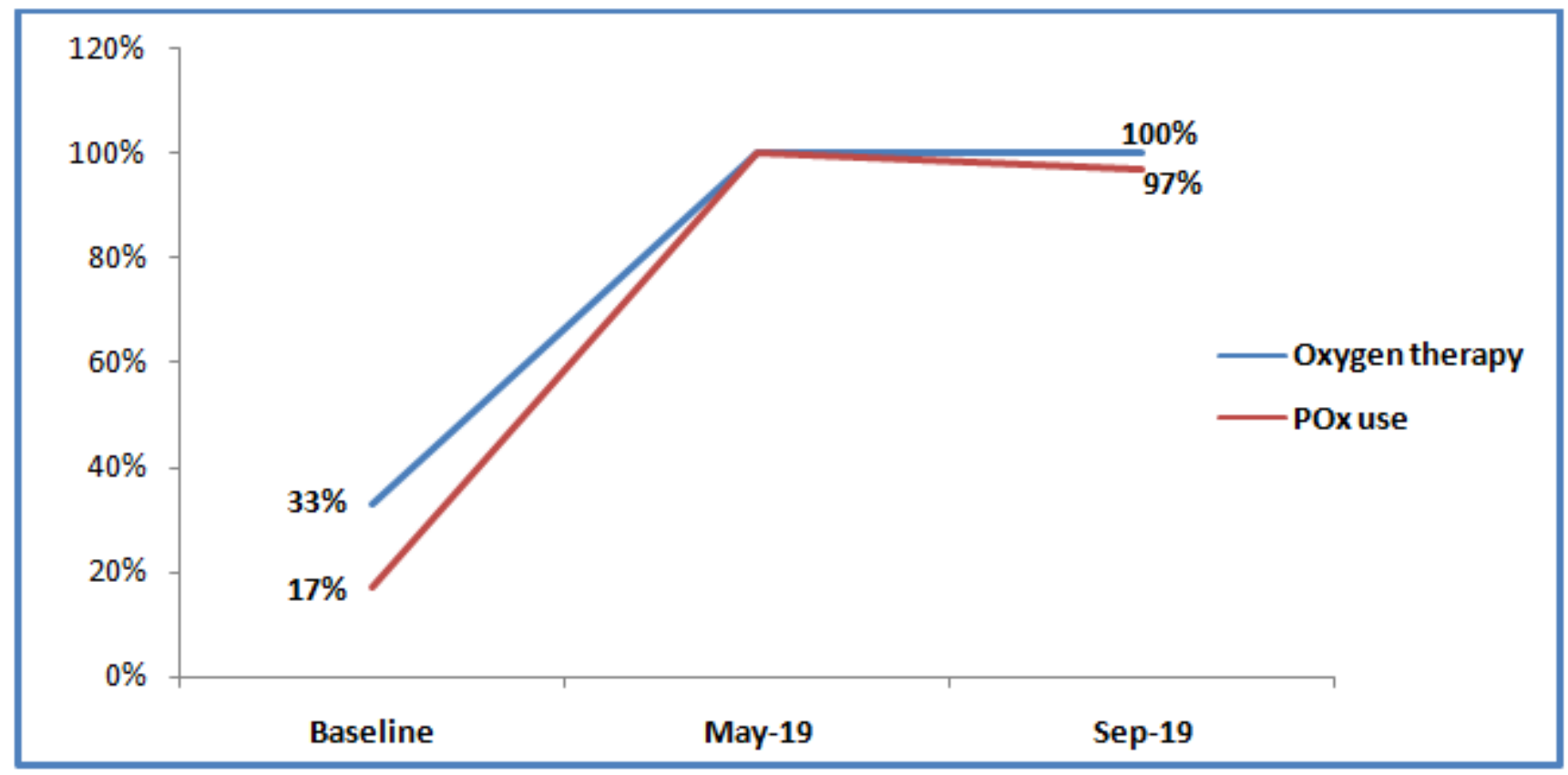

Figure 3

Trends in pulse oximetry use and oxygen therapy provision

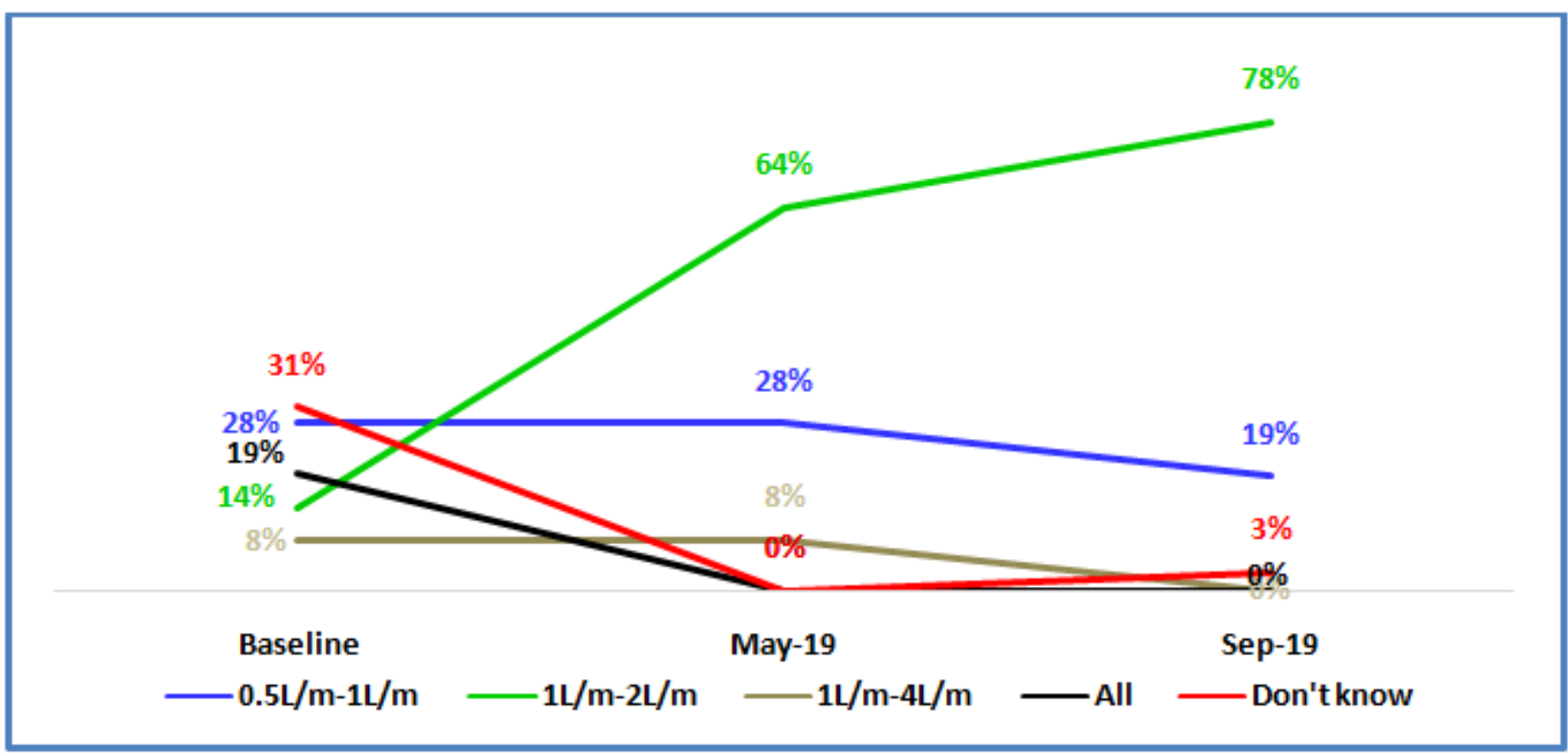

\section{Figure 4}

Knowledge of HCWs about the standard flow rate of oxygen administration for infants 


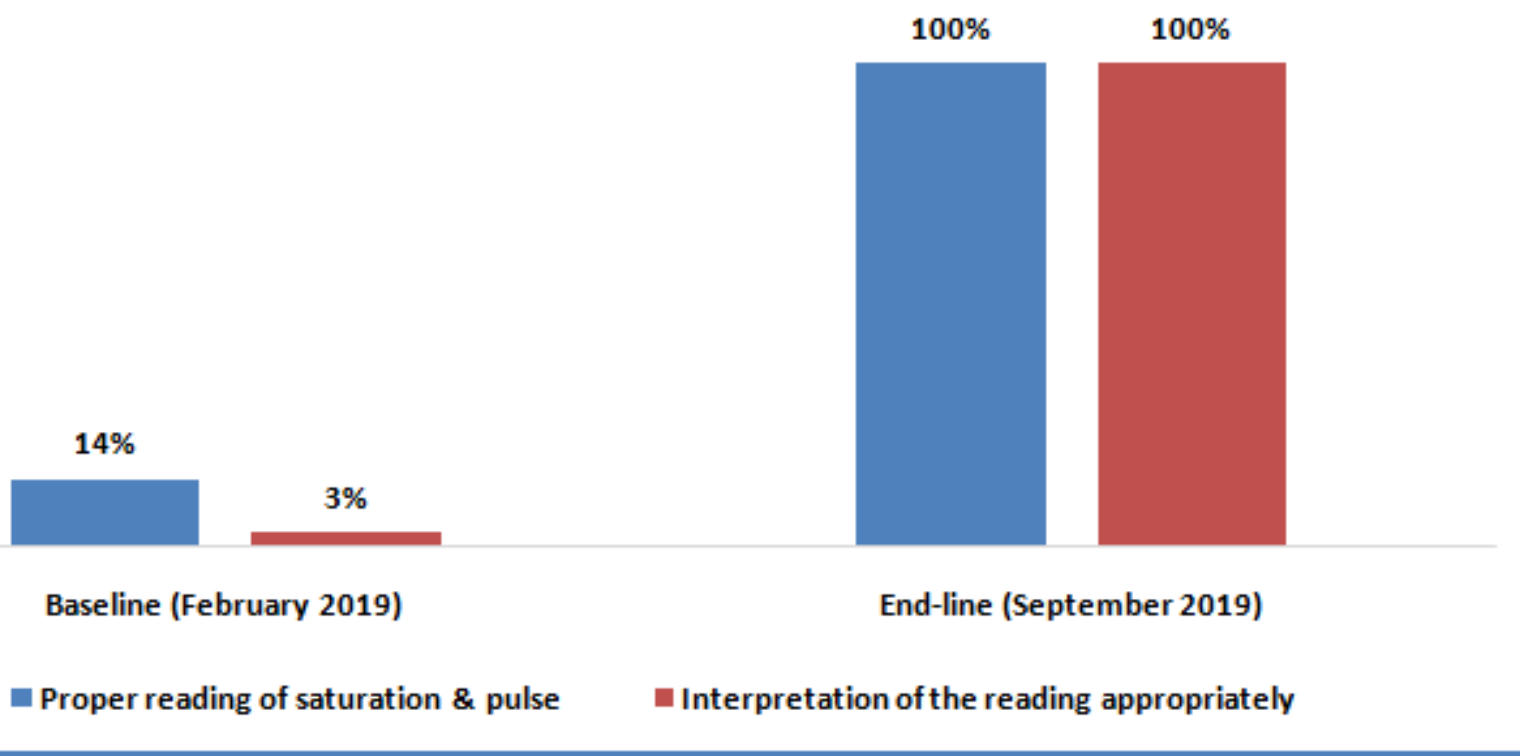

\section{Figure 5}

Proper reading and interpretation of the reading of saturation and pulse at baseline consecutive followups.

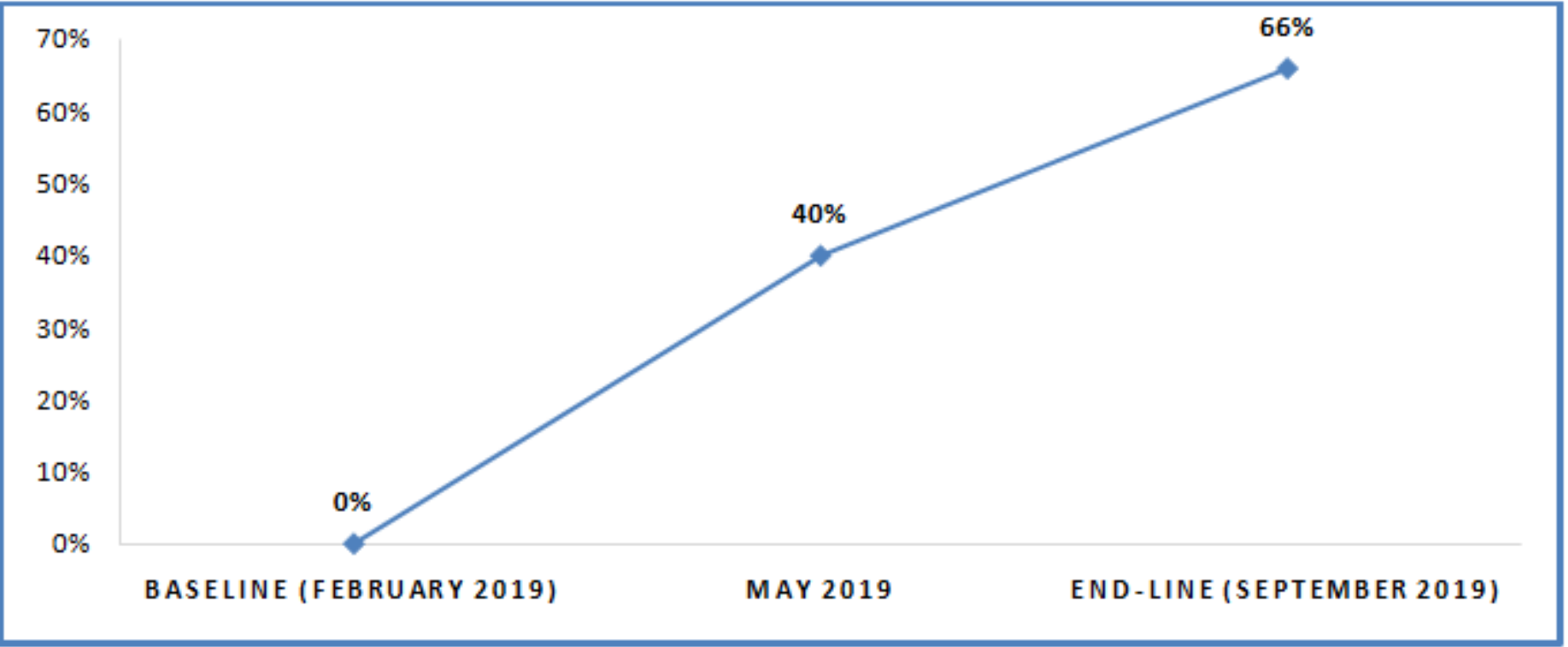

Figure 6

Oxygen saturation (SpO2) records at baseline, consecutive follow-ups 


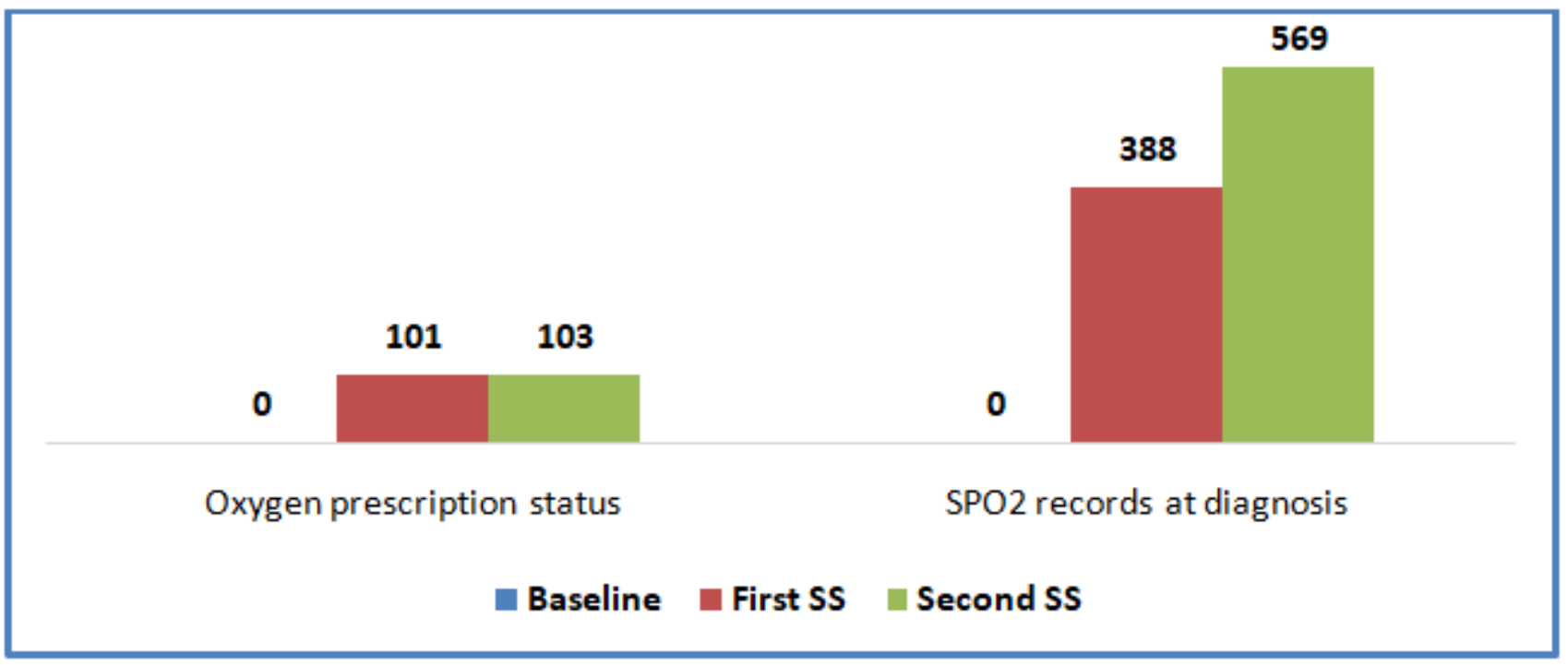

\section{Figure 7}

Status of oxygen prescription and SPO2 records at diagnosis (at triage and/or at initial assessment)

\section{Supplementary Files}

This is a list of supplementary files associated with this preprint. Click to download.

- 1.HCWSkillAssesBaseline.xlsx

- 1.HCMRRTOOLBaseline.xIsx

- 2.HCWKnowledgeAsses1stRound.xlsx

- 2.HCWSkillAsses1stRound.xIsx

- 2.HCMRRTOOL1stRound.xlsx

- 3.HCWKnowledgeAsses2ndRound.xlsx

- 3.HCWSkillAsses2ndRound.xlsx

- 3.HCMRRTOOL2ndRound.xIsx

- ChecklistforChartReview.docx

- Sectionl.1HealthCenterProfile.docx

- Sectionl.2HCWs.docx

- Sectionl.3HCWSkill.docx

- Sectionl.4Medicalequipmentmgt.docx

- Sectionl.5MedicalrecordreviewTool.docx 